\title{
Extendability of quadratic modules over a polynomial extension of an equicharacteristic regular local ring
}

\author{
A. A. Ambily ${ }^{\mathrm{a}}$, Ravi A. Rao ${ }^{\mathrm{b}}$ \\ ${ }^{a}$ Statistics and Mathematics Unit, Indian Statistical Institute, \\ Bangalore 560 059, India \\ ${ }^{b}$ School of Mathematics, Tata Institute of Fundamental Research, \\ Homi Bhabha Road, Mumbai 400 005, India
}

Dedicated to late Professor Amit Roy

\begin{abstract}
We prove that a quadratic $A[T]$-module $Q$ with Witt index $(Q / T Q) \geq d$, where $d$ is the dimension of the equicharacteristic regular local ring $A$, is extended from $A$. This improves a theorem of the second named author who showed it when $A$ is the local ring at a smooth point of an affine variety over an infinite field. To establish our result, we need to establish a Local-Global Principle (of Quillen) for the Dickson-Siegel-Eichler-Roy (DSER) elementary orthogonal transformations.
\end{abstract}

Keywords. Quadratic modules, Dickson-Siegel-Eichler-Roy transformations, Local-Global Principle, Extendability.

AMS Subject classifications (2010). 19G99, 13C10, 11E70, 20H25

\section{Introduction}

Let $A$ be a commutative Noetherian ring in which 2 is invertible and let $B$ be the polynomial $A$-algebra $A\left[X_{1}, \ldots, X_{n}\right]$ in $n$ indeterminates. Let $Q=(Q, q)$ be a quadratic space over $B$ and let $Q_{0}=\left(Q_{0}, q_{0}\right)$ be the reduction of $Q$ modulo the ideal of $B$ generated by $X_{1}, \ldots, X_{n}$. In [19], A. A. Suslin and V. I. Kopeiko proved that if $Q$ is stably extended from $A$ and for every maximal ideal $\mathfrak{m}$ of $A$, the Witt index of $A_{\mathfrak{m}} \otimes_{A}\left(Q_{0}, q_{0}\right)$ is larger than the Krull dimension of $A$, then $(Q, q)$ is extended from $A$. A shorter proof of this, due to Inta Bertuccioni, can be found in [6] and another proof is in the thesis of the second named author.

In the thesis of the second named author (see [13], [14]), it was shown that one can improve this result to Witt index $\geq d$, when $A$ is a local ring at a non-singular point of an affine variety of dimension $d$ over an infinite field. Moreover, a question was posed at the end of the thesis whether extendability can be shown for quadratic spaces with Witt index $\geq d$ over polynomial extensions of any equicharacteristic regular local ring of dimension $d$.

In this article, we establish this question affirmatively.

Email addresses: ambily@isibang.ac.in (A. A. Ambily), ravi@math.tifr.res.in (Ravi A. Rao) 
A few words on the proof: The analysis of the equicharacteristic regular local ring is done by a patching argument, akin to the one developed by Amit Roy in his paper [16]. This argument reduces the problem to the case of a complete equicharacteristic regular ring; which is a power series ring over a field, provided one can patch the information.

We found it useful to use Amit Roy's elementary orthogonal transformations in [15] for quadratic spaces with a hyperbolic summand over a commutative ring. These transformations (over fields) are known as Siegel transformations or Eichler transformations in the literature: we give a brief historical statement of the development.

These transformations (in matrix form) of quadratic spaces $(V, q)$ over finite fields first appeared on pg.12 in L. E. Dickson's book "Linear groups: With an exposition of the Galois field theory" (1958), which is an unaltered republication of the first edition (Teubner, Leipzig, 1901). Later in "Sur les groupes classiques" (1948), J. Dieudonné extended these results over infinite fields.

These orthogonal transformations (in a matrix form) over general fields also appeared in a paper of C. L. Siegel: Über die analytische Theorie der quadratischen Formen II. Annals of Math. 36 (1935), 230-263.

Another interpretation occurs in his work "Über die Zetafunktionen indefiniter quadratischer Formen", II., Math. Zeitschrift, 1938, 398-426 (on page 408). Here he used it to define the mass of representation of 0 by an indefinite quadratic form.

M. Eichler studied these transformations of $Q \perp H(k)$ in his study of the orthogonal group over fields $k$ and made the first systematic use of them in his famous book "Quadratische Formen und orthogonale Gruppen", first published in 1952, and reprinted in 1974.

(Eichler credits Siegel's 1935 paper for introducing these transformations in the notes on $\S 3$ on pg. 212 of his book, and also refers to the 1938 Zeitschrift paper of Siegel on pg. 218. He does not seem to be aware of Dickson's work.)

Amit Roy studied C. T. C. Wall's paper [21], who relied on Eichler's book. Amit Roy rewrote the transformations of Eichler in Wall's paper. He then generalized these transformations in his thesis (1967) over any commutative ring $R$. We shall call these the DSER elementary orthogonal transformations or just (Roy's) elementary orthogonal transformation group.

Note that these transformations of Roy have been further extended to form rings by L. N. Vaserstein (when the ring is local), and A. Bak (to the general case) in their thesis (respectively).

We show that the patching process is possible by establishing a Local-Global Principle for the Elementary Orthogonal group of a quadratic space with a hyperbolic summand. For this, we follow the broad outline of A. A. Suslin's method in [18] which led to a $K_{1}$ analogue of D. Quillen's Local-Global Principle in [12]. Instead of using Suslin's 'theory of generic forms which are elementary', we follow the more 'hands on' approach via the yoga of commutators. For this, we have to first find an appropriate generating set for Roy's group; which is the primary objective of $\S 2$ (That this set generates the group is proved in $\S 3$, via V. Suresh's lemma in [17]). We record the commutator calculus in $\S 4$, and refer the reader to rigorous proof of these identities to an article we have placed in the arXiv [1]. These commutator calculations enable us to prove the Local-Global Principle for Roy's group of orthogonal transformations over a polynomial extension.

As an interesting by-product, one realizes from the yoga of commutators in this elementary 
orthogonal group that it mimics Tang's well-known group in some features defined in [20], and the unitary group of Bass defined in [5]. The first named author intends to pursue the study of this group in more detail in a sequel article, where she hopes to establish A. Bak's type (see [4]) solvability theorem for the quotient group by the elementary subgroup.

Note: To make the reading effortless, we have placed the onerous (but straightforward) computations in this group as an article on the arXiv (see [1]) which can be accessed by any reader.

Finally, we have not attempted to study the ' $\Lambda$-ring variant' of this problem via the variant elementary orthogonal group as defined by A. Bak in his thesis (see [3]). We feel that it will throw more light on the interrelationship between all these groups; which will be carried out in a separate venture by the first named author.

\section{Preliminaries}

Let $A$ be a commutative ring in which 2 is invertible. A quadratic A-module is a pair $(M, q)$, where $M$ is an $A$-module and $q$ is a quadratic form on $M$. A quadratic space over $A$ is a pair $(M, q)$, where $M$ is a finitely generated projective $A$-module and $q: M \longrightarrow A$ is a nonsingular quadratic form. Let $M^{*}$ denotes the dual of the module $M$. Let $B_{q}$ be the symmetric bilinear form associated to $q$ on $M$, which is given by $B_{q}(x, y)=q(x+y)-q(x)-q(y)$ and $d_{B_{q}}: M \rightarrow M^{*}$ be the induced isomorphism given by $d_{B_{q}}(x)(y)=B_{q}(x, y)$, where $x, y \in M$. Given two quadratic $A$-modules $\left(M_{1}, q_{1}\right)$ and $\left(M_{2}, q_{2}\right)$, their orthogonal sum $(M, q)$ is defined by taking $M=M_{1} \oplus M_{2}$ and $q\left(\left(x_{1}, x_{2}\right)\right)=q_{1}\left(x_{1}\right)+q_{2}\left(x_{2}\right)$ for $x_{1} \in M_{1}, x_{2} \in M_{2}$. Denote $(M, q)$ by $\left(M_{1}, q_{1}\right) \perp\left(M_{2}, q_{2}\right)$ and $q$ by $q_{1} \perp q_{2}$.

Let $P$ be a finitely generated projective $A$-module. The module $P \oplus P^{*}$ has a natural quadratic form given by $p((x, f))=f(x)$ for $x \in P, f \in P^{*}$. The corresponding bilinear form $B_{p}$ is given by $B_{p}\left(\left(x_{1}, f_{1}\right),\left(x_{2}, f_{2}\right)\right)=f_{1}\left(x_{2}\right)+f_{2}\left(x_{1}\right)$ for $x_{1}, x_{2} \in P$ and $f_{1}, f_{2} \in P^{*}$. The quadratic space $\left(P \oplus P^{*}, p\right)$, denoted by $H(P)$, is called the hyperbolic space of $P$. A quadratic space $M$ is said to be hyperbolic if it is isometric to $H(P)$ for some $P$. The quadratic space $H(A)$, denoted by $h$, is called a hyperbolic plane. The orthogonal sum $h \perp h \perp \cdots \perp h$ of $n$ hyperbolic planes is denoted by $h^{n}$. A quadratic space $M$ is said to have Witt index $\geq n$ if $M \simeq M_{0} \perp H(P)$, where rank $P \geq n$. A quadratic space $M$ is said to have hyperbolic rank $\geq n$ if $M \simeq M_{0} \perp h^{d}$, where $d \geq n$. A quadratic space $M$ is said to be cancellative if for any quadratic $A$-spaces $M_{1}, M_{2}$ with $M \perp M_{2} \simeq M_{1} \perp M_{2}$, then $M \simeq M_{1}$.

Let $Q$ be a quadratic $A$-space and $P$ be a finitely generated projective $A$-module. Now let $M=Q \perp H(P)$. This is a quadratic space with the quadratic form $q \perp p$. The associated bilinear form on $M$, denoted by $\langle\cdot, \cdot\rangle$, is given by

$$
\langle(a, x),(b, y)\rangle=B_{q}((a, b))+B_{p}((x, y)) \text { for all } a, b \in Q \text { and } x, y \in H(P)
$$

where $B_{q}$ and $B_{p}$ are the bilinear forms on $Q$ and $P$. Let $M=M(B, q)$ be a quadratic module over $A$ with quadratic form $q$ and associated symmetric bilinear form $B$. Then the orthogonal group of $M$ is defined as follows:

$$
\varnothing_{A}(M)=\{\sigma \in \operatorname{Aut}(M) \mid q(\sigma(x))=q(x) \text { for all } x \in M\},
$$

where $\operatorname{Aut}(M)$ be the group of all $A$-linear automorphisms of $M$. 
Let $M$ be a free module of finite rank. By choosing a basis for $M$, we can define

$$
\mathrm{SO}_{A}(M)=\mathrm{SL}(M) \cap \varnothing_{A}(M),
$$

where $\operatorname{SL}(M)$ is the subgroup of $\operatorname{Aut}(M)$ consists of automorphisms of determinant 1. This is a normal subgroup of $\varnothing_{A}(M)$ and is called the special orthogonal group of $M$. See [7] for more details.

For any $A$-linear map $\alpha: Q \rightarrow P\left(\beta: Q \rightarrow P^{*}\right)$, the dual map $\alpha^{t}: P^{*} \rightarrow Q^{*}\left(\beta^{t}: P^{* *} \simeq\right.$ $\left.P \rightarrow Q^{*}\right)$ is defined as $\alpha^{t}(\varphi)=\varphi \circ \alpha\left(\beta^{t}\left(\varphi^{*}\right)=\varphi^{*} \circ \beta\right)$ for $\varphi \in P^{*}\left(\varphi^{*} \in P^{* *}\right)$. Recall from [15], the $A$-linear map $\alpha^{*}: P^{*} \rightarrow Q\left(\beta^{*}: P \rightarrow Q\right)$ is defined by $\alpha^{*}=d_{B_{q}}^{-1} \circ \alpha^{t}\left(\beta^{*}=d_{B_{q}}^{-1} \circ \beta^{t} \circ \varepsilon\right.$, where $\varepsilon: P \rightarrow P^{* *}$ is the natural isomorphism) and is characterized by the relation

$$
(f \circ \alpha)(z)=B_{q}\left(\alpha^{*}(f), z\right) \text { for } f \in P^{*}, z \in Q .
$$

In [15], A. Roy defined the "elementary" transformations $E_{\alpha}, E_{\beta}^{*}$ of $Q \perp H(P)$ given by

$$
\begin{array}{ll}
E_{\alpha}(z)=z+\alpha(z) & E_{\beta}^{*}(z)=z+\beta(z) \\
E_{\alpha}(x)=x & E_{\beta}^{*}(x)=-\beta^{*}(x)+x-\frac{1}{2} \beta \beta^{*}(x) \\
E_{\alpha}(f)=-\alpha^{*}(f)-\frac{1}{2} \alpha \alpha^{*}(f)+f & E_{\beta}^{*}(f)=f
\end{array}
$$

for $z \in Q, x \in P$ and $f \in P^{*}$. Observe that these transformations are orthogonal with respect to the above quadratic form $q \perp p$.

Now we recall the notion of generalized dimension function from [10]. Let $\mathcal{P} \subset \operatorname{Spec} A$ be a set of primes, $\mathbb{N}$ be the set of natural numbers and $d: \mathcal{P} \rightarrow \mathbb{N} \cup\{0\}$ be a function. For primes $\mathfrak{p}, \mathfrak{q}$ of $\mathcal{P}$, define a partial order $\ll$ on $\mathcal{P}$ as $\mathfrak{p} \ll \mathfrak{q}$ iff $\mathfrak{p} \subset \mathfrak{q}$ and $d(\mathfrak{p})>d(\mathfrak{q})$. A function $d: \mathcal{P} \rightarrow \mathbb{N} \cup\{0\}$ is a generalized dimension function if for any ideal $I$ of $A, V(I) \cap \mathcal{P}$ has only a finite number of minimal elements with respect to the partial ordering $\ll$.

We found it difficult to give a meaningful set of commutator relations for the set of generators $\left\{E_{\alpha}, E_{\beta}^{*} \mid \alpha \in \operatorname{Hom}_{A}(Q, P), \beta \in \operatorname{Hom}_{A}\left(Q, P^{*}\right)\right\}$.

Let $Q$ and $P$ be free $A$-modules. In this case, we could conceive of a natural set of generators, for which we could develop the commutator machinery. These generators will be denoted by $E_{\alpha_{i j}}, E_{\beta_{i j}}^{*}$ below. We proceed to define these now.

Notation 2.1. Let $P$ and $Q$ be free modules of rank $m$ and $n$ respectively, then we can identify $P, P^{*}$ and $Q$ with $A^{m}, A^{m}$ and $A^{n}$ respectively. Let $\left\{z_{i}: 1 \leq i \leq n\right\}$ be a basis for $Q,\left\{g_{i}: 1 \leq i \leq n\right\}$ be a basis for $Q^{*},\left\{x_{i}: 1 \leq i \leq m\right\}$ be a basis for $P$ and $\left\{f_{i}: 1 \leq i \leq m\right\}$ be a basis for $P^{*}$.

Let $p_{i}: A^{n} \longrightarrow A$ be the projection onto the $i^{\text {th }}$ component and $\eta_{i}: A \longrightarrow A^{n}$ be the inclusion into the $i^{\text {th }}$ component. Let $\alpha \in \operatorname{Hom}(Q, P)$. Let $\alpha_{i}, \alpha_{i j} \in \operatorname{Hom}(Q, P)$ be the maps given by

$$
\alpha_{i}=\eta_{i} \circ p_{i} \circ \alpha \quad \text { and } \quad \alpha_{i j}=\eta_{i} \circ p_{i} \circ \alpha \circ \eta_{j} \circ p_{j}
$$

for $1 \leq i \leq m$ and $1 \leq j \leq n$. Clearly $\alpha=\sum_{i=1}^{m} \alpha_{i}=\sum_{i=1}^{m} \sum_{j=1}^{n} \alpha_{i j}$. Then $\alpha_{i}^{*}, \alpha_{i j}^{*} \in \operatorname{Hom}\left(P^{*}, Q\right)$ is the maps given by

$$
\alpha_{i}^{*}=\left(\alpha^{*}\right)_{i}=\alpha^{*} \circ \eta_{i} \circ p_{i} \quad \text { and } \quad \alpha_{i j}^{*}=\left(\alpha^{*}\right)_{i j}=\eta_{j} \circ p_{j} \circ \alpha^{*} \circ \eta_{i} \circ p_{i} .
$$


Then $\alpha^{*}=\sum_{i=1}^{m} \alpha_{i}^{*}=\sum_{i=1}^{m} \sum_{j=1}^{n} \alpha_{i j}^{*}$. One can also see that this definition of $\alpha_{i}^{*}$, $\alpha_{i j}^{*}$ coincides with the one obtained by applying $\alpha^{*}=d_{B_{q}}{ }^{-1} \circ \alpha^{t} \in \operatorname{Hom}\left(P^{*}, Q\right)$ to $\alpha_{i}$ and $\alpha_{i j}$.

Let $z=\sum_{j=1}^{n} d_{j} z_{j} \in Q$ for $d_{j} \in A(1 \leq j \leq n)$. Then $\alpha$ is given by $\alpha\left(z_{j}\right)=x^{(j)}=\sum_{i=1}^{m} b_{i j} x_{i}$ for $b_{i j} \in A(1 \leq i \leq m)$ and $\alpha(z)=\sum_{j=1}^{n} \sum_{i=1}^{m} d_{j} b_{i j} x_{i}, \alpha_{i}(z)=\sum_{j=1}^{n} d_{j} b_{i j} x_{i}$ and $\alpha_{i j}(z)=d_{j} b_{i j} x_{i}$. Let $\alpha^{*}\left(f_{i}\right)=w_{i}$ for some $w_{i} \in Q$. If $f=\sum_{i=1}^{m} c_{i} f_{i}$ for $c_{i} \in A(1 \leq i \leq m)$, then $c_{i}=\left\langle f, x_{i}\right\rangle$ and so $\alpha^{*}(f)=\sum_{i=1}^{m}\left\langle f, x_{i}\right\rangle w_{i}$. If $w_{i}=\sum_{j=1}^{n} y_{j} z_{j}$ for some $y_{j} \in A$, then $w_{i j}=y_{j} z_{j} \in Q$.

For $1 \leq i \leq m$ and $1 \leq j \leq n$, the maps $\alpha_{i}^{*}$ and $\alpha_{i j}^{*}$ 's are given by

$$
\alpha_{i}^{*}\left(f_{j}\right)=\left\{\begin{array}{ll}
w_{i} & \text { if } \quad j=i, \\
0 & \text { if } \quad j \neq i .
\end{array} \quad \alpha_{i j}^{*}\left(f_{k}\right)=\left\{\begin{array}{lll}
w_{i j} & \text { if } \quad k=i, \\
0 & \text { if } \quad k \neq i .
\end{array}\right.\right.
$$

Let $\beta \in \operatorname{Hom}\left(Q, P^{*}\right)$. Set $\beta^{*}\left(x_{i}\right)=v_{i}$ for some $v_{i} \in Q$, let $v_{i j}$ denotes the element $\eta_{j} \circ p_{j}\left(v_{i}\right)$. Now defining the maps $\beta_{i}, \beta_{i j}, \beta_{i}^{*}, \beta_{i j}^{*}$ similarly and extending these to the whole of $Q \oplus P \oplus P^{*}$, we get the maps as follows: For $z \in Q, x \in P, f \in P^{*} ; 1 \leq i \leq m$ and $1 \leq j \leq n$;

$$
\begin{array}{lll}
\alpha_{i j}(z, x, f)=\left(0,\left\langle w_{i j}, z\right\rangle x_{i}, 0\right), & \beta_{i j}(z, x, f)=\left(0,0,\left\langle v_{i j}, z\right\rangle f_{i}\right), \\
\alpha_{i}(z, x, f)=\left(0,\left\langle w_{i}, z\right\rangle x_{i}, 0\right), & \beta_{i}(z, x, f)=\left(0,0,\left\langle v_{i}, z\right\rangle f_{i}\right), \\
\alpha(z, x, f)=\left(0, \Sigma_{i=1}^{m}\left\langle w_{i}, z\right\rangle x_{i}, 0\right), & & \beta(z, x, f)=\left(0,0, \Sigma_{i=1}^{m}\left\langle v_{i}, z\right\rangle f_{i}\right), \\
\alpha_{i j}^{*}(z, x, f)=\left(\left\langle f, x_{i}\right\rangle w_{i j}, 0,0\right), & \beta_{i j}^{*}(z, x, f)=\left(\left\langle x, f_{i}\right\rangle v_{i j}, 0,0\right), \\
\alpha_{i}^{*}(z, x, f)=\left(\left\langle f, x_{i}\right\rangle w_{i}, 0,0\right), & \beta_{i}^{*}(z, x, f)=\left(\left\langle x, f_{i}\right\rangle v_{i}, 0,0\right), \\
\alpha^{*}(z, x, f)=\left(\sum_{i=1}^{m}\left\langle f, x_{i}\right\rangle w_{i}, 0,0\right), & \beta^{*}(z, x, f)=\left(\sum_{i=1}^{m}\left\langle x, f_{i}\right\rangle v_{i}, 0,0\right) .
\end{array}
$$

Also, $q\left(w_{i j}\right)=\frac{1}{2}\left\langle w_{i j}, w_{i j}\right\rangle$ and $q\left(v_{i j}\right)=\frac{1}{2}\left\langle v_{i j}, v_{i j}\right\rangle$.

For $\alpha \in \operatorname{Hom}(Q, P)$, the orthogonal transformation $E_{\alpha_{i j}}$ on $Q \perp H(P)$ is given by

$$
\begin{aligned}
E_{\alpha_{i j}}(z, x, f) & =\left(I-\alpha_{i j}^{*}+\alpha_{i j}-\frac{1}{2} \alpha_{i j} \alpha_{i j}^{*}\right)(z, x, f) \\
& =\left(z-\left\langle f, x_{i}\right\rangle w_{i j}, x+\left\langle w_{i j}, z\right\rangle x_{i}-\left\langle f, x_{i}\right\rangle q\left(w_{i j}\right) x_{i}, f\right) .
\end{aligned}
$$

For $\beta \in \operatorname{Hom}\left(Q, P^{*}\right)$, the orthogonal transformation $E_{\beta_{i j}}^{*}$ of $Q \perp H(P)$ is given by

$$
\begin{aligned}
E_{\beta_{i j}}^{*}(z, x, f) & =\left(I-\beta_{i j}^{*}+\beta_{i j}-\frac{1}{2} \beta_{i j} \beta_{i j}^{*}\right)(z, x, f) \\
& =\left(z-\left\langle f_{i}, x\right\rangle v_{i j}, x, f+\left\langle v_{i j}, z\right\rangle f_{i}-\left\langle x, f_{i}\right\rangle q\left(v_{i j}\right) f_{i}\right) .
\end{aligned}
$$

\section{Roy's Elementary orthogonal transformations}

In this section, we consider the orthogonal group of $Q \perp H(P)$, denoted by $\varnothing_{A}(Q \perp$ $H(P)$ ), where $Q$ and $P$ are free $A$-modules of finite rank. Precisely,

$\varnothing_{A}(Q \perp H(P))=\{\sigma \in \operatorname{Aut}(Q \perp H(P)) \mid(q \perp p)(\sigma(z, y))=(q \perp p)(z, y) \forall(z, y) \in Q \perp H(P)\}$.

Since $Q$ and $P$ are free modules, the elements of $\varnothing_{A}(Q \perp H(P))$ can be represented as matrices over $A$ by choosing a basis for $Q$ and $P$. Then we can identify $\varnothing_{A}(Q \perp H(P))$ as a subgroup of $\mathrm{GL}_{(n+2 m)}(A)$. 
Lemma 3.1. An $(n+2 m) \times(n+2 m)$ matrix $T=\left(\begin{array}{ccc}A & B & C \\ D & F & G \\ H & J & K\end{array}\right)$ belongs to $\varnothing_{A}(Q \perp H(P))$ if and only if any of the following equations hold.

(a) $T^{t} \psi T=\psi$, for $\psi=\left(\begin{array}{ccc}\phi & 0 & 0 \\ 0 & 0 & I \\ 0 & I & 0\end{array}\right)$, where $\phi$ is the matrix corresponding to the nonsingular quadratic form $q$ on $Q$ and $\left(\begin{array}{ll}0 & I \\ I & 0\end{array}\right)$ is the matrix of the hyperbolic form $p$.

(b) $\left(\begin{array}{ccc}\phi^{-1} A^{t} \phi & \phi^{-1} H^{t} & \phi^{-1} D^{t} \\ C^{t} \phi & K^{t} & G^{t} \\ B^{t} \phi & J^{t} & F^{t}\end{array}\right) \cdot\left(\begin{array}{ccc}A & B & C \\ D & F & G \\ H & J & K\end{array}\right)=I d$.

Proof. Follows immediately from the definition of $\emptyset_{A}(Q \perp H(P))$.

Let $\mathrm{EO}_{A}(Q \perp H(P))$ be the subgroup of $\varnothing_{A}(Q \perp H(P))$ generated by $E_{\alpha}$ and $E_{\beta}^{*}$, where $\alpha \in \operatorname{Hom}(Q, P)$ and $\beta \in \operatorname{Hom}\left(Q, P^{*}\right)$. We call this group elementary orthogonal group and these transformations elementary orthogonal transformations. If $Q$ and $P$ are free modules of rank $n$ and $m$ respectively, we have the elementary transformations of the type $E_{\alpha_{i j}}$ and $E_{\beta_{i j}}^{*}$ for $1 \leq i \leq m, 1 \leq j \leq n$.

Next, we compare the elementary orthogonal group of Roy's elementary transformations and that of the Dickson-Siegel-Eichler transformations which is defined as follows:

Definition 3.2. [7, Chapter 5] Let $(M, B, q)$ be a non-degenerate quadratic module over $A$ and let $\varnothing_{A}(M)$ be its orthogonal group. Let $u$ and $v$ be in $M$ with $u$ isotropic and $B(u, v)=0$. For $r=q(v)$, define the Dickson-Siegel-Eichler transformation $\Sigma_{u, v, r} \in \operatorname{End}(M)$, by

$$
\Sigma_{u, v, r}(x)=x+u B(v, x)-v B(u, x)-u r B(u, x) .
$$

One can easily verify the following properties of Eichler transformations.

(i) $\Sigma_{u, v, q(v)} \in \emptyset_{A}(M)$,

(ii) $\Sigma_{u, v, q(v)} \Sigma_{u, w, q(w)}=\Sigma_{u, v+w, q(v)+q(w)+h(v, w)}$,

(iii) $\Sigma_{u, v, q(v)}^{-1}=\Sigma_{u,-v, q(v)}$,

(iv) $\sigma \Sigma_{u, v, q(v)} \sigma^{-1}=\Sigma_{\sigma u, \sigma v, q(v)}$ for $\sigma \in \varnothing_{A}(M)$.

Observe that $\Sigma_{0,0,0}=I d$.

We may regard the elementary orthogonal transformations $E_{\alpha_{i j}}$ and $E_{\beta_{i j}}^{*}$ as DicksonSiegel-Eichler transformations. More precisely, the orthogonal transformation $E_{\alpha_{i j}}$ of $M=$ $Q \perp H(P)$ given by

$$
E_{\alpha_{i j}}(z, x, f)=\left(z-\left\langle f, x_{i}\right\rangle w_{i j}, x+\left\langle w_{i j}, z\right\rangle x_{i}-\left\langle f, x_{i}\right\rangle q\left(w_{i j}\right) x_{i}, f\right)
$$


can be written as $\Sigma_{x_{i}, w_{i j}, q\left(w_{i j}\right)}(z, x, f)$. For,

$$
\begin{aligned}
\Sigma_{x_{i}, w_{i j}, q\left(w_{i j}\right)}(z, x, f)= & (z, x, f)+\left(0, x_{i}, 0\right)\left\langle\left(w_{i j}, 0,0\right),(z, x, f)\right\rangle-\left(w_{i j}, 0,0\right) \\
& \left\langle\left(0, x_{i}, 0\right),(z, x, f)\right\rangle-\left(0, x_{i}, 0\right) q\left(w_{i j}\right)\left\langle\left(0, x_{i}, 0\right),(z, x, f)\right\rangle \\
= & \left(z-\left\langle f, x_{i}\right\rangle w_{i j}, x+\left\langle w_{i j}, z\right\rangle x_{i}-\left\langle f, x_{i}\right\rangle q\left(w_{i j}\right) x_{i}, f\right) .
\end{aligned}
$$

Similarly, the orthogonal transformation $E_{\beta_{i j}}^{*}$ of $M$ given by

$$
E_{\beta_{i j}}^{*}(z, x, f)=\left(z-\left\langle f_{i}, x\right\rangle v_{i j}, x, f+\left\langle v_{i j}, z\right\rangle f_{i}-\left\langle x, f_{i}\right\rangle q\left(v_{i j}\right) f_{i}\right)
$$

can be written as $\Sigma_{f_{i}, v_{i j}, q\left(v_{i j}\right)}(z, x, f)$.

These elementary orthogonal transformations also satisfy the properties listed above. Moreover, as we saw in the previous section, they satisfy a more general set of properties analogous to Property(ii).

The transformations defined by A. Roy [15] can also be viewed as unitary transvections [5, Section 5] of certain types of quadratic modules over a unitary ring $(A, \lambda, \Lambda)$. See [5, Section 4] for further details of unitary rings.

Let $M=V \perp H(P)$. If $x=(v ; p, q) \in M$, we have $f(x, x)=f(v, v)+\langle q, p\rangle_{P}$. Suppose $P$ has a unimodular element $p_{0}$. i.e. there is a $q_{0} \in \bar{P}$ such that $\left\langle q_{0}, p_{0}\right\rangle_{P}=1$. For any elements $p_{0} \in P, w_{0} \in V$ and $a_{0} \in A$ with $a_{0} \equiv f\left(w_{0}, w_{0}\right) \bmod \Lambda$,

$$
\begin{aligned}
f\left(p_{0}, p_{0}\right) & \in \Lambda, \\
\left\langle w_{0}, p_{0}\right\rangle & =0, \\
f\left(w_{0}, w_{0}\right) & \equiv a_{0} \bmod \Lambda .
\end{aligned}
$$

If $x=(v ; p, q)$, then

$$
\sigma_{p_{0}, a_{0}, w_{0}}(x)=x+p_{0}\left\langle w_{0}, x\right\rangle-w_{0} \bar{\lambda}\left\langle p_{0}, x\right\rangle-p_{0} \bar{\lambda} a_{0}\left\langle p_{0}, x\right\rangle
$$

Now take $\Lambda=0, \lambda=1, f\left(w_{0}, w_{0}\right)=a_{0}$ and $\left\langle w_{0}, w_{0}\right\rangle=2 f\left(w_{0}, w_{0}\right)=2 a_{0}$. Then we get

$$
\begin{aligned}
& E_{\alpha_{i j}}(z, x, f)=\sigma_{x_{i}, \frac{\left\langle w_{i j}, w_{i j}\right\rangle}{2}, w_{i j}}(z, x, f), \\
& E_{\beta_{i j}}^{*}(z, f, x)=\sigma_{f_{i}, \frac{\left\langle v_{i j}, v_{i j}\right\rangle}{2}, v_{i j}}(z, f, x) .
\end{aligned}
$$

Now we state the splitting property and extend the Lemma 1.4 of [17] regarding Roy's transformations. We use the notation $E(\alpha)$ for either $E_{\alpha}$ or $E_{\alpha}^{*}$, where $\alpha \in \operatorname{Hom}(Q, P)$ or $\operatorname{Hom}\left(Q, P^{*}\right)$ respectively. Combining Lemma 1.2 and Lemma 1.3 of [17], we have the following:

Lemma 3.3 (Splitting property [17]). For $\alpha_{1}, \alpha_{2} \in \operatorname{Hom}(Q, P)$ or $\operatorname{Hom}\left(Q, P^{*}\right)$ we have

$$
E\left(\alpha_{1}+\alpha_{2}\right)=E\left(\frac{\alpha_{1}}{2}\right) E\left(\alpha_{2}\right) E\left(\frac{\alpha_{1}}{2}\right)=E\left(\frac{\alpha_{2}}{2}\right) E\left(\alpha_{1}\right) E\left(\frac{\alpha_{2}}{2}\right) .
$$

The following lemma extends Lemma 1.4 of [17].

Lemma 3.4. With the notation as above, the group $\mathrm{EO}_{A}(Q \perp H(P))$ is generated by $E\left(\alpha_{i j}\right)$, with $\alpha \in \operatorname{Hom}(Q, P)$ or $\operatorname{Hom}\left(Q, P^{*}\right) ; 1 \leq i \leq m$ and $1 \leq j \leq n$. 
Proof. For $\alpha \in \operatorname{Hom}(Q, P)$ or $\operatorname{Hom}\left(Q, P^{*}\right)$, we have $\alpha=\sum_{i=1}^{m} \sum_{j=1}^{n} \alpha_{i j}$ from the previous section. By repeated applications of the splitting property, we have

$$
\begin{aligned}
E(\alpha)= & E\left(\frac{\alpha_{11}}{2}\right) E\left(\frac{\alpha_{21}}{2}\right) \cdots E\left(\frac{\alpha_{m 1}}{2}\right) E\left(\frac{\alpha_{12}}{2}\right) \cdots E\left(\frac{\alpha_{m 2}}{2}\right) \\
& \cdots E\left(\frac{\alpha_{(m-1) n}}{2}\right) E\left(\alpha_{m n}\right) E\left(\frac{\alpha_{(m-1) n}}{2}\right) \cdots E\left(\frac{\alpha_{11}}{2}\right) .
\end{aligned}
$$

This proves the lemma.

\section{Commutator relations between elementary generators}

All the main results in this paper depend on various commutator relations between the generators of $\mathrm{EO}_{A}(Q \perp H(P))$. The computations for these relations are messy and the general expressions and their detailed proofs are given in a note posted in the arXiv at [1].

In this section, we state some of the commutator relations which will be used in the sections to follow.

Lemma 4.1. Let $Q, P$ be free $A$-modules of rank $n$ and $m$ respectively; $\alpha, \delta \in \operatorname{Hom}(Q, P)$ and $\beta, \gamma \in \operatorname{Hom}\left(Q, P^{*}\right)$. Then for any given $i, j, k, l$ with $i \neq k$ for $1 \leq i, k \leq m$ and $1 \leq j, l \leq n$; we have the following commutator relations between the elementary orthogonal transformations $E_{\alpha_{i j}}, E_{\delta_{k l}}, E_{\beta_{k l}}^{*}$ and $E_{\gamma_{k l}}^{*}$ :

(i) $\left[E_{\alpha_{i j}}, E_{\delta_{k l}}\right]=I+\delta_{k l} \alpha_{i j}^{*}-\alpha_{i j} \delta_{k l}^{*}$,

(ii) $\left[E_{\alpha_{i j}}, E_{\beta_{k l}}^{*}\right]=I-\alpha_{i j} \beta_{k l}^{*}+\beta_{k l} \alpha_{i j}^{*}$,

(iii) $\left[E_{\beta_{i j}}^{*}, E_{\gamma_{k l}}^{*}\right]=I+\gamma_{k l} \beta_{i j}^{*}-\beta_{i j} \gamma_{k l}^{*}$.

Proof. (i), (ii) and (iii) follows from Lemma 2.1, Lemma 2.3 and Lemma 2.7 of [1] respectively.

Corollary 4.2. Under the same assumptions as in Lemma 4.1 and for $a, b, c, d \in A$, we have the following:

(i) $\left[E_{a \alpha_{i j}}, E_{b \delta_{k l}}\right]=\left[E_{c \alpha_{i j}}, E_{d \delta_{k l}}\right]$ if $a b=c d$,

(ii) $\left[E_{a \alpha_{i j}}, E_{b \beta_{k l}}^{*}\right]=\left[E_{c \alpha_{i j}}, E_{d \beta_{k l}}^{*}\right]$ if $a b=c d$,

(iii) $\left[E_{a \beta_{i j}}^{*}, E_{b \gamma_{k l}}^{*}\right]=\left[E_{c \beta_{i j}}^{*}, E_{d \gamma_{k l}}^{*}\right]$ if $a b=c d$.

Proof. (i), (ii) and (iii) follows from Corollary 2.2, Corollary 2.5 and Corollary 2.8 of [1] respectively.

Lemma 4.3. Let $Q, P$ be free $A$-modules of rank $n$ and $m$ respectively; $\alpha, \delta \in \operatorname{Hom}(Q, P)$ and $\beta, \gamma \in \operatorname{Hom}\left(Q, P^{*}\right)$. For any given $i, j, k, l, p, q$ with $i \neq k, k \neq p$ for $1 \leq i, k, p \leq m$ and $1 \leq j, l, q \leq n$; we have the following commutator relations:

(i) $\left[E_{\beta_{i j}}^{*},\left[E_{\alpha_{k l}}, E_{\delta_{p q}}\right]\right]=E_{\lambda_{k j}}\left[E_{\beta_{i j}}^{*}, E_{\frac{\lambda_{k j}}{2}}\right]$, 
(ii) $\left[E_{\alpha_{i j}},\left[E_{\delta_{k l}}, E_{\beta_{p q}}^{*}\right]\right]=E_{\mu_{k j}}\left[E_{\alpha_{i j}}, E_{\frac{\mu_{k j}}{2}}\right]$,

(iii) $\left[E_{\beta_{i j}}^{*},\left[E_{\gamma_{k l}}^{*}, E_{\alpha_{p q}}\right]\right]=E_{\nu_{k j}}^{*}\left[E_{\beta_{i j}}^{*}, E_{\frac{\nu_{k j}}{2}}^{*}\right]$,

(iv) $\left[E_{\alpha_{i j}},\left[E_{\beta_{k l}}^{*}, E_{\gamma_{p q}}^{*}\right]\right]=E_{\xi_{k j}}^{*}\left[E_{\alpha_{i j}}, E_{\frac{\xi_{k j}}{2}}^{*}\right]$,

where

$$
\begin{gathered}
\lambda_{k j}=\alpha_{k l} \delta_{p q}^{*} \beta_{i j}, \mu_{k j}=\delta_{k l} \beta_{p q}^{*} \alpha_{i j} \in \operatorname{Hom}(Q, P), \\
\nu_{k j}=\gamma_{k l} \alpha_{p q}^{*} \beta_{i j} \text { and } \xi_{k j}=\beta_{k l} \gamma_{p q}^{*} \alpha_{i j} \in \operatorname{Hom}\left(Q, P^{*}\right) .
\end{gathered}
$$

Proof. (i), (ii), (iii) and (iv) follows from Lemma 3.1, Lemma 3.3, Lemma 3.5 and Lemma 3.7 of [1] respectively.

Corollary 4.4. Under the same assumptions as in Lemma 4.3 and for $a, b, c, d, e, f \in A$, we have the following:

(i) $\left[E_{a \beta_{i j}}^{*},\left[E_{b \alpha_{k l}}, E_{c \delta_{p q}}\right]\right]=\left[E_{d \beta_{i j}}^{*},\left[E_{e \alpha_{k l}}, E_{f \delta_{p q}}\right]\right]$ if abc=def and $a^{2} b c=d^{2} e f$,

(ii) $\left[E_{a \alpha_{i j}},\left[E_{b \delta_{k l}}, E_{c \beta_{p q}}^{*}\right]\right]=\left[E_{d \alpha_{i j}},\left[E_{e \delta_{k l}}, E_{f \beta_{p q}}^{*}\right]\right]$ if $a b c=\operatorname{def}$ and $a^{2} b c=d^{2} e f$,

(iii) $\left[E_{a \beta_{i j}}^{*},\left[E_{b \gamma_{k l}}^{*}, E_{c \alpha_{p q}}\right]\right]=\left[E_{d \beta_{i j}}^{*},\left[E_{e \gamma_{k l}}^{*}, E_{f \alpha_{p q}}\right]\right]$ if $a b c=\operatorname{def}$ and $a^{2} b c=d^{2} e f$,

(iv) $\left[E_{a \alpha_{i j}},\left[E_{b \beta_{k l}}^{*}, E_{c \gamma_{p q}}^{*}\right]\right]=\left[E_{d \alpha_{i j}},\left[E_{e \beta_{k l}}^{*}, E_{f \gamma_{p q}}^{*}\right]\right]$ if $a b c=\operatorname{def}$ and $a^{2} b c=d^{2} e f$.

Proof. (i), (ii), (iii) and (iv) follows from Corollary 3.2, Corollary 3.4, Corollary 3.6 and Corollary 3.8 of [1] respectively.

\section{Local-Global Principle for Roy's Elementary Orthogonal Transformations}

In this section, we establish that $E O_{A[X]}(M[X])$, where $M=Q \perp H(P)$ such that $Q$ and $P$ are free modules of rank $n$ and $m$ respectively, satisfies the Local-Global principle.

Theorem 5.1 (Local-Global Principle). Let $\theta(X) \in \varnothing_{A[X]}(M[X])$. If for all maximal ideals $\mathfrak{m}$ of $A, \theta(X)_{\mathfrak{m}} \in \varnothing_{A_{\mathfrak{m}}}\left(M_{\mathfrak{m}}\right) \cdot \mathrm{EO}_{A_{\mathfrak{m}}[X]}\left(M_{\mathfrak{m}}[X]\right)$, then $\theta(X) \in \varnothing_{A}(M) \cdot \mathrm{EO}_{A[X]}(M[X])$.

Before beginning the proof we make the following remark.

Remark 5.2. Replacing $\theta(X)$ by $\theta(0)^{-1} \theta(X)$, we may assume that $\theta(0)=1$. Further for any ring $A, \theta(X) \in \varnothing_{A}(M) \mathrm{EO}_{A[X]}(M[X])$ and $\theta(0)=I d$ implies that $\theta(X) \in \mathrm{EO}_{A[X]}(M[X])$.

For, if $\theta(X)=\gamma \varepsilon(X), \gamma \in \varnothing_{A}(M)$ and $\varepsilon(X) \in \mathrm{EO}_{A[X]}(M[X])$, then $\gamma=\theta(0) \varepsilon(0)^{-1}=\varepsilon(0)^{-1}$.

In view of this remark we can rewrite the Theorem 5.1 as follows:

Theorem 5.3 (Local-Global Principle). Let $\theta(X) \in \varnothing_{A[X]}(M[X])$ with $\theta(0)=$ Id. If for all maximal ideals $\mathfrak{m}$ of $A, \theta(X)_{\mathfrak{m}} \in E O_{A_{\mathfrak{m}}[X]}\left(M_{\mathfrak{m}}[X]\right)$, then $\theta(X) \in \mathrm{EO}_{A[X]}(M[X])$. 
We begin with some lemmas.

Lemma 5.4. Let $G$ be a group and $a_{i}, b_{i} \in G$, for $i=1, \ldots, n$. Then

$$
\prod_{i=1}^{n} a_{i} b_{i}=\prod_{i=1}^{n} r_{i} b_{i} r_{i}^{-1} \prod_{i=1}^{n} a_{i}
$$

where $r_{i}=\prod_{j=1}^{i} a_{j}$.

Proof. Direct computation.

Lemma 5.5. The group $\mathrm{EO}_{A[X]}(M[X])$ is generated by the elements of the type $\gamma E\left(X \alpha_{i j}(X)\right) \gamma^{-1}$, where $\gamma \in \operatorname{EO}_{A}(M), \alpha_{i j}(X) \in \operatorname{Hom}(Q[X], P[X])$ or $\operatorname{Hom}\left(Q[X], P^{*}[X]\right)$.

Proof. Let $\theta(X)$ be an element of $\mathrm{EO}_{A[X]}(M[X])$ such that $\theta(0)=I d$. Then

$$
\begin{aligned}
\theta(X) & =\prod_{k=1}^{r} E\left(\alpha_{i_{k} j_{k}}(X)\right)=\prod_{k=1}^{r} E\left(\alpha_{i_{k} j_{k}}(0)+X \alpha_{i_{k} j_{k}}^{\prime}(X)\right) \\
& =\prod_{k=1}^{r} E\left(\frac{\alpha_{i_{k} j_{k}}(0)}{2}\right) E\left(X \alpha_{i_{k} j_{k}}^{\prime}(X)\right) E\left(\frac{\alpha_{i_{k} j_{k}}(0)}{2}\right)(\text { by Splitting property ) } \\
& =\prod_{k=1}^{r+1} a_{k} b_{k},
\end{aligned}
$$

where

$$
\begin{array}{lll}
a_{1}=E\left(\frac{\alpha_{i_{1} j_{1}}(0)}{2}\right), & b_{k}=E\left(X \alpha_{i_{k} j_{k}}^{\prime}(X)\right) \text { for } k=1, \ldots, r, \\
a_{k}=E\left(\frac{\alpha_{i_{k-1} j_{k-1}}(0)}{2}\right) E\left(\frac{\alpha_{i_{k} j_{k}}(0)}{2}\right) & \text { for } k=2, \ldots, r, \\
a_{r+1}=E\left(\frac{\alpha_{i_{r} j_{r}}(0)}{2}\right), & b_{r+1}=1 .
\end{array}
$$

By Lemma 5.4, we have

$$
\theta(X)=\prod_{k=1}^{r+1} \gamma_{k} E\left(X \alpha_{i_{k} j_{k}}^{\prime}(X)\right) \gamma_{k}^{-1} \prod_{k=1}^{r+1} a_{k},
$$

where $\gamma_{k}=\prod_{j=1}^{k} a_{j} \in \mathrm{EO}_{A}(M)$ and $\prod_{k=1}^{r+1} a_{k}=\prod_{k=1}^{r} E\left(\alpha_{i_{k} j_{k}}(0)\right)=\theta(0)=I d$. Therefore

$$
\theta(X)=\prod_{k=1}^{r+1} \gamma_{k} E\left(X \alpha_{i_{k} j_{k}}^{\prime}(X)\right) \gamma_{k}^{-1}
$$

Lemma 5.6. Let $\alpha, \delta \in \operatorname{Hom}(Q, P), \beta, \gamma \in \operatorname{Hom}\left(Q, P^{*}\right)$ and $s$ be a non-nilpotent element of A. Fix $r \in \mathbb{N}$. Let $i, k, p_{t} \in\{1,2, \ldots, m\}$ and $j, l, q_{t} \in\{1,2, \ldots, n\}$ for every $t \in \mathbb{N}$. Then for sufficiently large $d$, there exists a product decomposition for $E\left(\frac{a}{s^{r}} X_{i j}\right) E\left(s^{d} x Y_{k l}\right) E\left(-\frac{a}{s^{r}} X_{i j}\right)$ in $\mathrm{EO}_{A_{s}}\left(M_{s}\right)$ given by

$$
E\left(\frac{a}{s^{r}} X_{i j}\right) E\left(s^{d} x Y_{k l}\right) E\left(-\frac{a}{s^{r}} X_{i j}\right)=\prod_{t=1}^{\nu} E\left(s^{d_{t}} x_{t} Z_{p_{t} q_{t}}\right),
$$

where $X, Y, Z \in\{\alpha, \beta, \gamma, \delta\}, a, x \in A$ and the elements $x_{t} \in A$ for $t \in \mathbb{N}$ are chosen suitably. 
Proof. To prove the lemma it is enough to consider the following cases:

Case 1: $(X, Y) \in\{(\alpha, \alpha),(\alpha, \delta),(\beta, \beta),(\beta, \gamma)\}$

$$
E\left(\frac{a}{s^{r}} X_{i j}\right) E\left(s^{d} x Y_{k l}\right) E\left(\frac{a}{s^{r}} X_{i j}\right)^{-1}=\prod_{t=1}^{\nu} E\left(s^{d_{t}} x_{t} Z_{p_{t} q_{t}}\right) .
$$

Subcase (a): $i \neq k$

$$
\begin{aligned}
E\left(\frac{a}{s^{r}} X_{i j}\right) E\left(s^{d} x Y_{k l}\right) E\left(\frac{a}{s^{r}} X_{i j}\right)^{-1} & =\left[E\left(\frac{a}{s^{r}} X_{i j}\right), E\left(s^{d} x Y_{k l}\right)\right] E\left(s^{d} x Y_{k l}\right) \\
& =\left[E\left(a s^{p} X_{i j}\right), E\left(s^{q} x Y_{k l}\right)\right] E\left(s^{d} x Y_{k l}\right) \\
& \quad(\text { by Corollary 4.2 (i) and Corollary 4.2 (iii)) } \\
= & \prod_{t=1}^{\nu} E\left(s^{d_{t}} x_{t} Z_{p_{t} q_{t}}\right) \quad \text { for } d_{t}>0 .
\end{aligned}
$$

This equation holds for any positive integers $p, q$ with $p+q=d-r$.

Subcase (b): $i=k$

$$
\begin{aligned}
E\left(\frac{a}{s^{r}} X_{i j}\right) E\left(s^{d} x Y_{k l}\right) E\left(\frac{a}{s^{r}} X_{i j}\right)^{-1} & =\left[E\left(\frac{a}{s^{r}} X_{i j}\right), E\left(s^{d} x Y_{k l}\right)\right] E\left(s^{d} x Y_{k l}\right) \\
& =E\left(s^{d} x Y_{k l}\right) .
\end{aligned}
$$

(by Lemma 4.1(i) and by Lemma 4.1 (iii))

Case 2: $(X, Y) \in\{(\alpha, \beta),(\beta, \alpha)\}$

$$
E\left(\frac{a}{s^{r}} X_{i j}\right) E\left(s^{d} x Y_{k l}\right) E\left(\frac{a}{s^{r}} X_{i j}\right)^{-1}=\prod_{t=1}^{\nu} E\left(s^{d_{t}} x_{t} Z_{p_{t} q_{t}}\right) .
$$

Subcase (a): $i \neq k$

For instance,

$$
\begin{aligned}
E\left(\frac{a}{s^{r}} \alpha_{i j}\right) E\left(s^{d} x \beta_{k l}\right) E\left(\frac{a}{s^{r}} \alpha_{i j}\right)^{-1} & =E_{\frac{a}{s^{r}} \alpha_{i j}} E_{s^{d} x \beta_{k l}}^{*} E_{\frac{a}{s^{r}} \alpha_{i j}}^{-1} \\
& =\left[E_{\frac{a}{s^{r}} \alpha_{i j}}, E_{s^{d} x \beta_{k l}}^{*}\right] E_{s^{d} x \beta_{k l}}^{*} \quad \text { (by Corollary 4.2 (ii)) } \\
& =\left[E_{a s^{p} \alpha_{i j}}, E_{s^{q} x \beta_{k l}}^{*} E_{s^{d} x \beta_{k l}}^{*} \quad \text { for } d_{t}>0 \text { and } \nu \leq 5 .\right.
\end{aligned}
$$

Subcase (b): $i=k$

For instance,

$$
E\left(\frac{a}{s^{r}} \alpha_{i j}\right) E\left(s^{d} x \beta_{i l}\right) E\left(\frac{a}{s^{r}} \alpha_{i j}\right)^{-1}=E_{\frac{a}{s^{r}} \alpha_{i j}} E_{s^{d} x \beta_{i l}}^{*} E_{\frac{a}{s^{r}} \alpha_{i j}}^{-1} .
$$

Set $d=N_{1}+N_{2}+N_{3}$ such that $N_{1} \geq r+2$ and $N_{2}+N_{3} \geq 2 r+4$. Now, replacing $E_{s^{d} x \beta_{i l}}^{*}$ by $\left[E_{s^{N_{1}} \alpha_{k l}},\left[E_{s^{N_{2}} x \beta_{i l}^{*}}, E_{s^{N_{3}} \gamma_{p q}^{*}}\right]\right]\left[E_{s^{d} x \frac{\beta_{i l}^{*}}{2}}, E_{s^{N_{1}} \alpha_{k l}}\right]$ in Equation (15.1), using Lemma 4.3 (i), we have

$$
E_{\frac{a}{s^{r}} \alpha_{i j}} E_{s^{d} x \beta_{i l}}^{*} E_{\frac{a}{s^{r}} \alpha_{i j}}^{-1}=E_{\frac{a}{s^{r}} \alpha_{i j}}\left[E_{s^{N_{1}} \alpha_{k l}},\left[E_{s^{N_{2} x \beta_{i l}^{*}}}, E_{s^{N_{3}} \gamma_{p q}^{*}}\right]\right]\left[E_{s^{d} x \frac{\beta_{i l}^{*}}{2}}, E_{s^{N_{1}} \alpha_{k l}}\right] E_{\frac{a}{s^{r}} \alpha_{i j}}^{-1} .
$$

Then we will see that the following are in the required product form. 
(i) $E_{\frac{a}{s^{\tau}} \alpha_{i j}} E_{s^{N_{1}} \alpha_{k l}} E_{\frac{a}{s^{\tau}}}^{-1} \alpha_{i j}$,

(ii) $E_{\frac{a}{s^{r}} \alpha_{i j}}\left[E_{s^{N_{2} x \beta_{i l}^{*}}}, E_{s^{N_{3}} \gamma_{p q}^{*}}\right] E_{\frac{a}{s^{r}} \alpha_{i j}}^{-1}$,

(iii) $E_{\frac{a}{s^{r}} \alpha_{i j}}\left[E_{s^{d} x \frac{\beta_{i l}^{*}}{2}}, E_{s^{N_{1}} \alpha_{k l}}\right] E_{\frac{a}{s^{r}} \alpha_{i j}}^{-1}$.

$$
\text { For, (i) } \begin{aligned}
E_{\frac{a}{s^{r}} \alpha_{i j}} E_{s^{N_{1}} \alpha_{k l}} E_{\frac{a}{s^{r}} \alpha_{i j}}^{-1} & =\left[E_{\frac{a}{s^{r}} \alpha_{i j}}, E_{s^{N_{1}}} \alpha_{k l}\right] E_{s^{N_{1}} \alpha_{k l}} \\
& =\left[E_{a s^{p^{\prime}} \alpha_{i j}}, E_{s^{q^{\prime}} \alpha_{k l}}\right] E_{s^{N_{1}} \alpha_{k l}}(\text { by Corollary } 4.2(\mathrm{i})) \\
& =\prod_{t=1}^{\nu} E\left(s^{d_{t}} x_{t} Z_{p_{t} q_{t}}\right) \text { for } d_{t}>0 \text { and } \nu \leq 5 .
\end{aligned}
$$

This equation holds for any positive integers $p^{\prime}, q^{\prime}$ with $p^{\prime}+q^{\prime}=N_{1}-r$.

$$
\text { (ii) } \begin{aligned}
E_{\frac{a}{s^{r}} \alpha_{i j}}\left[E_{s^{N_{2}} x \beta_{i l}^{*}}, E_{s^{N_{3}} \gamma_{p q}^{*}}\right] E_{\frac{a}{s^{r}} \alpha_{i j}}^{-1} & =\left[E_{\frac{a}{s^{r}} \alpha_{i j}}\left[E_{s^{N_{2} x \beta_{i l}^{*}}}, E_{s^{N_{3}} \gamma_{p q}^{*}}\right]\right]\left[E_{s^{N_{2} x \beta_{i l}^{*}}}, E_{s^{N_{3}} \gamma_{p q}^{*}}\right] \\
& =\left[E_{s^{p^{\prime \prime}} \alpha_{i j}},\left[E_{s^{q^{\prime \prime}} x \beta_{i l}^{*}}, E_{s^{r^{\prime \prime}} \gamma_{p q}^{*}}\right]\right]\left[E_{s^{N_{2}} x \beta_{i l}^{*}}, E_{s^{N_{3}} \gamma_{p_{q}}^{*}}\right] \\
& =\prod_{t=1}^{\nu} E\left(s^{d_{t}} x_{t} Z_{p_{t} q_{t}}\right) \text { for } d_{t}>0 \text { and } \nu \leq 14 .
\end{aligned}
$$

This equation holds for any positive integers $p^{\prime \prime}, q^{\prime \prime}$ and $r^{\prime \prime}$ with $2 p^{\prime \prime}+q^{\prime \prime}+r^{\prime \prime}=N_{2}+N_{3}-2 r$.

$$
\text { (iii) } \begin{aligned}
E_{\frac{a}{s^{r}} \alpha_{i j}}\left[E_{s^{d} x \frac{\beta_{i l}^{*}}{2}}, E_{s^{N_{1}} \alpha_{k l}}\right] E_{\frac{a}{s^{r}} \alpha_{i j}}^{-1} & =\left[E_{\frac{a}{s^{r}} \alpha_{i j}},\left[E_{s^{d} x \frac{\beta_{i l}^{*}}{2}}, E_{s^{N_{1}} \alpha_{k l}}\right]\right]\left[E_{s^{d} \frac{\beta_{i l}^{*}}{2}}, E_{s^{N_{1}} \alpha_{k l}}\right] \\
= & {\left[E_{s^{p^{\prime \prime \prime}} \alpha_{i j}},\left[E_{s^{q^{\prime \prime \prime}} \frac{\beta_{i l}^{*}}{2}}, E_{s^{r^{\prime \prime \prime}} \alpha_{k l}}\right]\right]\left[E_{s^{d} \frac{\beta_{i l}^{*}}{2}}, E_{s^{N_{1}} \alpha_{k l}}\right] } \\
= & \prod_{t=1}^{\nu} E\left(s^{d_{t}} x_{t} Z_{p_{t} q_{t}}\right) \text { for } d_{t}>0 \text { and } \nu \leq 14 .
\end{aligned}
$$

This equation holds for any positive integers $p^{\prime \prime \prime}, q^{\prime \prime \prime}$ and $r^{\prime \prime \prime}$ with $2 p^{\prime \prime \prime}+q^{\prime \prime \prime}+r^{\prime \prime \prime}=N_{1}+d-2 r$. Hence Equation (5.1) is of the form $\prod_{t=1}^{\nu} E\left(s^{d_{t}} x_{t} Z_{p_{t} q_{t}}\right)$ for $d_{t}>0$ and $\nu \leq 52$.

Lemma 5.7. (Dilation Lemma) Let $A$ be a commutative ring and $Q, P$ be free modules of rank $n$ and $m$ respectively. Let $s$ be a non-nilpotent element of $A$ and $M=Q \perp H(P)$. Let $\theta(X) \in \varnothing_{A[X]}(M[X])$ with $\theta(0)=I d$. Let $Y, Z \in \operatorname{Hom}(Q, P)$ or $\operatorname{Hom}\left(Q, P^{*}\right)$. If $\theta_{s}(X)=(\theta(X))_{s} \in \mathrm{EO}_{A[X]_{s}}\left(M[X]_{s}\right)$, then for $d \gg 0$ and for all $b \in(s)^{d} A$, we have $\theta(b X) \in \mathrm{EO}_{A[X]}(M[X])$.

Proof. Let $\theta_{s}(X) \in \mathrm{EO}_{A_{s}[X]}\left(M_{s}[X]\right)$. Then $\theta_{s}(X)=\prod_{k=1}^{r} E\left(\alpha_{i_{k} j_{k}}(X)\right)$, where $\alpha_{i_{k} j_{k}}(X) \in \operatorname{Hom}\left(Q_{s}[X], P_{s}[X]\right)$ or $\operatorname{Hom}\left(Q_{s}[X], P_{s}^{*}[X]\right)$ for all $k \in \mathbb{N}, i_{k} \in\{1,2, \ldots, m\}$ and $j_{k} \in\{1,2, \ldots, n\}$.

Let $\alpha_{i_{k} j_{k}}(X)=\alpha_{i_{k} j_{k}}(0)+X \alpha_{i_{k} j_{k}}^{\prime}(X)$. By the splitting property, we can write

$$
E\left(\alpha_{i_{k} j_{k}}(X)\right)=E\left(\frac{\alpha_{i_{k} j_{k}}(0)}{2}\right) E\left(X \alpha_{i_{k} j_{k}}^{\prime}(X)\right) E\left(\frac{\alpha_{i_{k} j_{k}}(0)}{2}\right) .
$$




$$
\text { Then } \theta_{s}(X)=\prod_{k=1}^{r+1} E\left(\frac{\alpha_{i_{k} j_{k}}(0)}{2}\right) E\left(X \alpha_{i_{k} j_{k}}^{\prime}(X)\right) E\left(\frac{\alpha_{i_{k} j_{k}}(0)}{2}\right) .
$$

By Lemma 5.5, one has

$$
\theta_{s}(X)=\prod_{k=1}^{r+1} \gamma_{k} E\left(X \alpha_{i_{k} j_{k}}^{\prime}(X)\right) \gamma_{k}^{-1}
$$

where $\gamma_{k}=\prod_{j=1}^{k} a_{j}$ with

$$
\begin{array}{ll}
a_{1}=E\left(\frac{\alpha_{i_{1} j_{1}}(0)}{2}\right), & a_{r+1}=E\left(\frac{\alpha_{i_{r} j_{r}}(0)}{2}\right), \\
a_{k}=E\left(\frac{\alpha_{i_{k-1} j_{k-1}}(0)}{2}\right) E\left(\frac{\alpha_{i_{k} j_{k}}(0)}{2}\right) & \text { for } k=2, \ldots, r .
\end{array}
$$

Hence we can write

$$
\theta_{s}\left(s^{d} X\right)=\prod_{k=1}^{r+1} \gamma_{k} E\left(s^{d} X \alpha_{i_{k} j_{k}}^{\prime}\left(s^{d} X\right)\right) \gamma_{k}^{-1} \text { for } d \gg 0 .
$$

Claim : If $\xi=\prod_{j=1}^{k} E\left(c_{j}\right), c_{j} \in M_{s}$, then for $\xi E\left(s^{d} x Z_{i j}\right) \xi^{-1}$, we have a product decomposition given by

$$
\xi E\left(s^{d} x Z_{i j}\right) \xi^{-1}=\prod_{t=1}^{\lambda_{k}} E\left(s^{d_{t}} x_{t} Z_{i_{t} j_{t}}\right)
$$

with $d_{t} \rightarrow \infty$ for $d \gg 0, x_{t} \in A$.

Proof of the Claim. We do this by induction on $k$.

Let $\xi=\xi_{1} \xi_{2} \ldots \xi_{k}$, where $\xi_{i}=E\left(c_{i}\right)$. When $k=1$, by Lemma 5.6, we have a product decomposition

$$
\xi_{1} E\left(s^{d} x Z_{i j}\right) \xi_{1}^{-1}=\prod_{t=1}^{\lambda_{1}} E\left(s^{d_{t}} x_{t} Z_{i_{t} j_{t}}\right)
$$

with $d_{t} \rightarrow \infty$ for $d \gg 0$. Now assume that the result is true for $k-1$. i.e. we have

$$
\xi_{1} \xi_{2} \ldots \xi_{k-1} E\left(s^{d} x Z_{i j}\right)\left(\xi_{1} \xi_{2} \ldots \xi_{k-1}\right)^{-1}=\prod_{t=1}^{\lambda_{k-1}} E\left(s^{d_{t}} x_{t} Z_{i_{t} j_{t}}\right)
$$

with $d_{t} \rightarrow \infty$ for $d \gg 0$. Now by Lemma 5.6, we can write

$$
\xi_{k} E\left(s^{d} x Z_{i j}\right) \xi_{k}^{-1}=\prod_{t=1}^{\lambda_{k-1}} E\left(s^{d_{t}} x_{t} Z_{i_{t} j_{t}}\right)=\mu_{1} \mu_{2} \ldots \mu_{\lambda} \text { (say). }
$$

Hence we have

$$
\begin{aligned}
\left(\xi_{1} \xi_{2} \ldots \xi_{k-1} \xi_{k}\right) E\left(s^{d} x Z_{i j}\right)\left(\xi_{1} \xi_{2} \ldots \xi_{k-1}\right)^{-1}= & \left(\xi_{1} \xi_{2} \ldots \xi_{k-1}\right) \mu_{1} \mu_{2} \ldots \mu_{\lambda}\left(\xi_{1} \xi_{2} \ldots \xi_{k-1}\right)^{-1} \\
= & \left(\xi_{1} \xi_{2} \ldots \xi_{k-1}\right) \mu_{1}\left(\xi_{1} \xi_{2} \ldots \xi_{k-1}\right)^{-1}\left(\xi_{1} \xi_{2} \ldots \xi_{k-1}\right) \\
& \mu_{2}\left(\xi_{1} \xi_{2} \ldots \xi_{k-1}\right)^{-1} \ldots\left(\xi_{1} \xi_{2} \ldots \xi_{k-1}\right) \\
& \mu_{\lambda}\left(\xi_{1} \xi_{2} \ldots \xi_{k-1}\right)^{-1} .
\end{aligned}
$$

Now applying induction to each of the expressions $\xi_{1} \xi_{2} \ldots \xi_{k-1} \mu_{l}\left(\xi_{1} \xi_{2} \ldots \xi_{k-1}\right)^{-1}$ as $l$ varies from 1 to $\lambda$, we have a product decomposition as in Equation(5.2). Therefore we can write

$$
\theta_{s}\left(s^{d} X\right)=\prod_{k=1}^{r+1} \prod_{t=1}^{\lambda_{k}} E\left(s^{d_{t}} x_{t} Z_{i_{t} j_{t}}\right)
$$

for $d$ large enough. The terms $s^{d_{t}} x_{t}$ for $1 \leq t \leq \lambda_{k}$ is contained in $M[X]$ as required. Hence

$$
\theta(b X)=\prod_{k=1}^{r+1} \prod_{t=1}^{\lambda_{k}} E\left(s^{d_{t}} x_{t} Z_{i_{t} j_{t}}\right) \in \mathrm{EO}_{A[X]}(M[X])
$$

for all $b \in(s)^{d} A$. 
Proof of the Theorem 5.3. Let $\mathfrak{m}$ be a maximal ideal of $A$. Choose an element $s_{\mathfrak{m}}$ from $A \backslash \mathfrak{m}$ such that

$$
\theta(X)_{s_{\mathfrak{m}}} \in E O_{A_{s}[X]_{\mathfrak{m}}}\left(M_{s}[X]_{\mathfrak{m}}\right) .
$$

Define

$$
\kappa(X, Y)=\theta(X+Y) \theta(Y)^{-1} .
$$

Clearly $\kappa(X, Y)_{s_{\mathfrak{m}}} \in E O_{A_{s}[X, Y]_{\mathfrak{m}}}\left(M_{s}[X]_{\mathfrak{m}}\right)$ and $\kappa(0, Y)=I d$.

Now by applying Dilation Lemma with $A[Y]$ as the base ring, we get

$$
\kappa\left(b_{\mathfrak{m}} X, Y\right) \in \mathrm{EO}_{A[X, Y]}(M[X, Y]),
$$

where $b_{\mathfrak{m}} \in\left(s_{\mathfrak{m}}^{N}\right)$ for some $N \gg 0$.

Since $A$ is the ideal generated by $\left\{s_{\mathfrak{m}}\right\}_{\mathfrak{m} \in \operatorname{Max} A}$, there exists maximal ideals $\mathfrak{m}_{1}, \ldots, \mathfrak{m}_{r}$ and elements $s_{\mathfrak{m}_{i}} \in A \backslash \mathfrak{m}_{i}$ such that $A=\sum_{i=1}^{r}\left(s_{\mathfrak{m}_{i}}\right)$. Therefore

$$
A=\sum_{i=1}^{r}\left(s_{\mathfrak{m}_{i}}^{N_{i}}\right)
$$

for any $N_{i}>0$. Hence for $b_{\mathfrak{m}_{i}} \in\left(s_{\mathfrak{m}_{i}}^{N_{i}}\right)$ with $N_{i} \gg 0$, there exists elements $d_{1}, \ldots, d_{r} \in A$ satisfying

$$
\sum_{i=1}^{r} d_{i} b_{\mathfrak{m}_{i}}=1 .
$$

Observe that $\kappa\left(d_{i} b_{\mathfrak{m}_{i}} X, Y\right) \in \mathrm{EO}_{A[X, Y]}(M[X, Y])$ for $1 \leq i \leq r$.

$$
\begin{aligned}
\theta(X)= & \theta\left(\sum_{i=1}^{r} d_{i} b_{\mathfrak{m}_{i}} X\right) \theta\left(\sum_{i=2}^{r} d_{i} b_{\mathfrak{m}_{i}} X\right)^{-1} \theta\left(\sum_{i=2}^{r} d_{i} b_{\mathfrak{m}_{i}} X\right) \theta\left(\sum_{i=3}^{r} d_{i} b_{\mathfrak{m}_{i}} X\right)^{-1} \cdots \\
& \theta\left(d_{r-1} b_{\mathfrak{m}_{r-1}} X+d_{r} b_{\mathfrak{m}_{r}} X\right) \theta\left(d_{r} b_{\mathfrak{m}_{r}} X\right)^{-1} \theta\left(d_{r} b_{\mathfrak{m}_{r}} X\right) \\
= & \prod_{i=1}^{r-1} \kappa\left(d_{i} b_{\mathfrak{m}_{i}} X, T_{i}\right) \kappa\left(d_{r} b_{\mathfrak{m}_{r}} X, 0\right),
\end{aligned}
$$

where $T_{i}=\sum_{k=i+1}^{r} d_{k} b_{\mathfrak{m}_{k}} X$. Hence $\theta(X) \in \mathrm{EO}_{A[X]}(M[X])$.

5.1. A Local-Global principle for $\mathrm{EO}\left(Q \perp h^{m}\right) \cdot \varnothing\left(h^{m}\right)$

Theorem 5.8 (14], Theorem 2.5). Let $A$ be a ring with generalized dimension $\geq d$. Let $(Q, q)$ be a diagonalizable quadratic A-space. Consider the quadratic A-space $Q \perp H(P)$, where $\operatorname{rank}(P)>d$. Then

$$
\begin{aligned}
\emptyset_{A}(Q \perp H(P)) & =\mathrm{EO}_{A}(Q, H(P)) \cdot \emptyset_{A}(H(P)) \\
& =\left\{\varepsilon \beta \mid \varepsilon \in \mathrm{EO}_{A}(Q, H(P)), \beta \in \emptyset_{A}(H(P))\right\} \\
& =\left\{\beta \varepsilon \mid \varepsilon \in \mathrm{EO}_{A}(Q, H(P)), \beta \in \emptyset_{A}(H(P))\right\} \\
& =\varnothing_{A}(H(P)) \cdot \mathrm{EO}_{A}(Q, H(P)) .
\end{aligned}
$$

Lemma 5.9. (Dilation Lemma) Let $A$ be a commutative ring with generalized dimension $\geq d$ and $Q$ be a free module of rank $n$. Let $(Q, q)$ be a diagonalizable quadratic A-space. Let $s$ be a non-nilpotent element of $A$ and $m>d$. Let $\theta(X) \in \varnothing_{A[X]}\left(Q \otimes A[X] \perp h^{m}\right) \cdot \varnothing_{A[X]}\left(h^{m}\right)$ with $\theta(0)=$ Id. If $\theta_{s}(X)=(\theta(X))_{s} \in \mathrm{EO}_{A[X]_{s}}\left(Q \otimes A[X]_{s} \perp h^{m}\right) \cdot \emptyset_{A[X]_{s}}\left(h^{m}\right)$, then for $d \gg 0$ and for all $b \in(s)^{d} A$, we have $\theta(b X) \in \mathrm{EO}_{A[X]}\left(Q \otimes A[X] \perp h^{m}\right) \cdot \varnothing_{A[X]}\left(h^{m}\right)$. 
Proof. The proof is similar to Lemma 5.7. For, if $\theta_{s}(X)=\varepsilon(X) \beta(X)$ with $\varepsilon(X) \in \mathrm{EO}_{A[X]_{s}}\left(Q \otimes A[X]_{s} \perp h^{m}\right), \beta(X) \in \varnothing_{A[X]_{s}}\left(h^{m}\right)$, then $\theta(0)=I=\varepsilon(0) \beta(0)$; whence $\theta_{s}(X)=\left\{\varepsilon(X) \varepsilon(0)^{-1}\right\}\left\{\beta(0)^{-1} \beta(X)\right\}$. In other words, we may assume at the onset that $\varepsilon(0)=I d$ and $\beta(0)=I d$. The rest of the proof follows from Lemma 5.7 .

Theorem 5.10. (Local-Global Principle) Let $A$ be a commutative ring with generalized dimension $\geq d$ and let $(Q, q)$ be a diagonalizable quadratic A-space. Assume that $Q$ is a free module of rank $n$. Let $m>d$ and let $\theta(X) \in \varnothing_{A[X]}\left(Q \otimes A[X] \perp h^{m}\right)$ with $\theta(0)=I d$. If $\forall \mathfrak{m} \in \operatorname{Max}(A), \alpha_{\mathfrak{m}}=\beta_{\mathfrak{m}} \gamma_{\mathfrak{m}}$, where $\beta_{\mathfrak{m}} \in \mathrm{EO}_{A[X]_{\mathfrak{m}}}\left((Q \otimes A[X])_{\mathfrak{m}} \perp h^{m}\right), \gamma_{\mathfrak{m}} \in \varnothing_{A[X]_{\mathfrak{m}}}\left(h^{m}\right)$ with $\beta(0)=I d, \gamma(0)=I d$. Then $\alpha=\beta \gamma$ with $\beta \in \mathrm{EO}_{A[X]}\left((Q \otimes A[X]) \perp h^{m}\right), \gamma \in \emptyset_{A[X]}\left(h^{m}\right)$.

Proof. The proof follows in similar lines as Theorem 5.3 except for the following. Let $\mathfrak{m}$ be a maximal ideal of $A$. Choose an element $s_{\mathfrak{m}}$ from $A \backslash \mathfrak{m}$ such that

$$
\theta(X)_{s_{\mathfrak{m}}} \in E O_{A[X]_{s_{\mathfrak{m}}}}\left(Q \otimes A[X]_{s_{\mathfrak{m}}} \perp h^{m}\right) \varnothing_{A[X]_{s_{\mathfrak{m}}}}\left(h^{m}\right) .
$$

Define

$$
\kappa(X, Y)=\theta(X+Y) \theta(Y)^{-1} .
$$

Then

$$
\kappa(X, Y)=\varepsilon_{1} \eta_{1} \varepsilon_{2} \eta_{2}
$$

for $\varepsilon_{1}, \varepsilon_{2} \in E O_{A[X, Y]}\left(Q \otimes A[X, Y] \perp h^{m}\right)$ and $\eta_{1}, \eta_{2} \in \varnothing_{A[X, Y]}\left(h^{m}\right)$. Since

$$
\mathrm{EO}_{A[X, Y]}\left(Q \otimes A[X, Y] \perp h^{m}\right) \cdot \varnothing_{A[X, Y]}\left(h^{m}\right)=\varnothing_{A[X, Y]}\left(h^{m}\right) \cdot \mathrm{EO}_{A[X, Y]}\left(Q \otimes A[X, Y] \perp h^{m}\right),
$$

by Theorem 5.8, we can write Equation (5.3) as

$$
\kappa(X, Y)=\varepsilon_{1} \varepsilon_{2}^{\prime} \eta_{1}^{\prime} \eta_{2}
$$

for some $\varepsilon_{2}^{\prime} \in \mathrm{EO}_{A[X, Y]}\left(Q \otimes A[X, Y] \perp h^{m}\right)$ and $\eta_{1}^{\prime} \in \varnothing_{A[X, Y]}\left(h^{m}\right)$.

Then $\kappa(X, Y)_{s_{\mathrm{m}}} \in E O_{A[X, Y]_{s_{\mathrm{m}}}}\left(Q \otimes A[X, Y]_{s_{\mathrm{m}}} \perp h^{m}\right) \cdot \varnothing_{A[X, Y]_{s_{\mathrm{m}}}}\left(h^{m}\right)$ and $\kappa(0, Y)=I d$.

Therefore, by applying Lemma 5.9 with base ring $A[Y]$,

$$
\kappa\left(b_{\mathfrak{m}} X, Y\right) \in \mathrm{EO}_{A[X, Y]}\left(Q \perp A[X, Y] \perp h^{m}\right) \cdot \varnothing_{A[X, Y]}\left(h^{m}\right),
$$

where $b_{\mathfrak{m}} \in\left(s_{\mathfrak{m}}^{N}\right)$ for some $N \gg 0$.

\section{Extendability of Quadratic Spaces}

In this section, we apply the Local Global principle to prove the principal result [Theorem 6.2 on the extendability of quadratic $A[T]$-spaces of Witt index $\geq d$ over an equicharacteristic regular local ring of dimension $d$.

We begin with the following crucial observation.

Lemma 6.1. Let $A$ be a regular local ring containing a field. Let $(Q, q) \perp h$ be a quadratic $A[T]$-space. If $(Q / T Q \perp h)$ is hyperbolic, then $(Q, q) \perp h$ is hyperbolic. 
Proof. In [11], D. Popescu showed that if $A$ is a geometrically regular local ring (over a field $k$ ), or when the characteristic of the residue field is a regular parameter in $A$, then it is a filtered inductive limit of regular local rings essentially of finite type over the integers (or over $k$ ).

In view of this, we may regard $(Q, q) \perp h$ to be a quadratic $B[T]$-space over some regular local ring B essentially of finite type over $k$ with $(Q / T Q, q /(T)) \perp h$ hyperbolic. In view of Proposition 1.3 of [13], $(Q, q) \perp h$ is hyperbolic over $B[T]$, whence over $A[T]$.

Theorem 6.2. Let $(A, \mathfrak{m})$ be an equicharacteristic regular local ring of dimension $d$ and $2 \in A^{*}$. Then every quadratic $A[T]$-space $(Q, q) \perp h^{n}$ with Witt index $n \geq d$ is extended from A.

Proof. Let $\left\{\pi_{1}, \pi_{2}, \ldots, \pi_{d}\right\}$ be a regular system of parameters generating the maximal ideal $\mathfrak{m}$ of $A$.

Let $A^{l}$ denote the $\left(\pi_{1}, \ldots, \pi_{l}\right)$-adic completion of $A$. Observe that $A^{d}$ is isomorphic to the power series ring $k\left[\left[X_{1}, \ldots, X_{d}\right]\right]$ by Cohen structure theorem, where $k$ is the residue field $A / \mathfrak{m}$ of $A$. Observe also that $A^{l}$ is the $\left(\pi_{l}\right)$-adic completion of $A^{l-1}$.

We now recall Amit Roy's garland of patching diagrams in [16]:

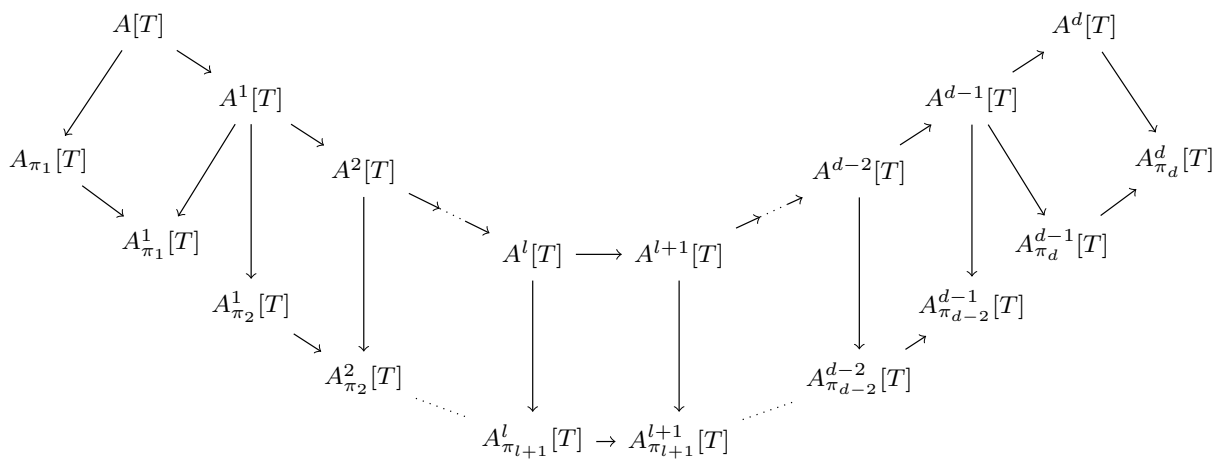

We use it below. Let us concentrate on the patching square $\mathcal{P}_{l}(A)[T]$ :

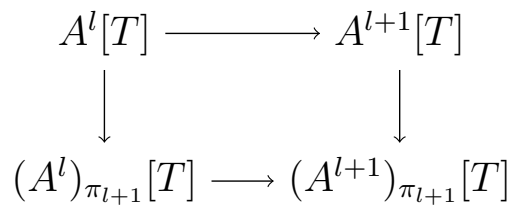

For all $l$, this is a cartesian square as rings. Moreover, by [9], it is also a cartesian square of quadratic spaces. This will enable us to analyze the quadratic $A$-space.

We prove the result by induction on $d-l$, starting with $l=0$. In this case $A$ is a complete equicharacteristic regular local ring, whence a power series ring over its residue field. We appeal to [13, Theorem 1.1].

Assume the result for $d-l=m$. For $d-(l+1)=m-1$, consider the patching diagram $\mathcal{P}_{m-1}(A)[T]$.

We fix some notations as folows:

For a regular parameter $\pi$ of $A$, let $Q^{l}=Q \otimes A^{l}[T], Q^{0}=Q, Q_{\pi}^{l}=Q \otimes A_{\pi}^{l}[T]$ and for a quadratic $A$-space $Q_{1}$, we denote $Q_{1} \otimes A^{l}$ by $Q_{1}^{l}$. 
Let $\left(Q \perp h^{n}\right) /(T)=Q_{1} \perp h^{n}$, where $Q_{1}$ is the quadratic $A$-space $Q /(T)$. Since $A^{m-1}$ is local, $Q_{1}{ }^{m-1}$ is diagonalizable [2, Proposition 3.4]. Since $A^{m-1}$ is regular, by Karoubi's theorem [8, Chapter VII, Theorem 2.1], $\left(Q \perp h^{n}\right)^{m-1}$ is stably extended from $A^{m-1}$. Let

$$
\left(Q \perp h^{n}\right)^{m-1} \perp h^{r} \stackrel{\simeq}{\longrightarrow} A^{m-1}[T] \otimes\left(Q_{1}{ }^{m-1} \perp h^{n+r}\right), n \geq d .
$$

Then

$$
\left(\left(Q \perp h^{n}\right)^{m-1} \perp h^{r}\right)_{\pi_{m}} \stackrel{\simeq}{\longrightarrow}\left(\left(A^{m-1}\right)_{\pi_{m}}[T] \otimes\left(\left(Q_{1}{ }^{m-1}\right)_{\pi_{m}} \perp h^{n+r}\right)\right), n \geq d .
$$

By [14, Theorem 3.3], we get the isomorphism

$$
\left(\left(Q \perp h^{n}\right)^{m-1}\right)_{\pi_{m}} \stackrel{\sigma}{\longrightarrow}\left(\left(A^{m-1}\right)_{\pi_{m}}[T] \otimes\left(\left(Q_{1}{ }^{m-1}\right)_{\pi_{m}} \perp h^{n}\right)\right) .
$$

Using the extendability for quadratic spaces over $A^{m}[T]$ via induction hypothesis, we have

$$
\tau:\left(Q \perp h^{n}\right)^{m} \stackrel{\simeq}{\longrightarrow} A^{m}[T] \otimes\left(Q_{1}{ }^{m} \perp h^{n}\right) .
$$

Now by identifying the quadratic spaces $\left(\left(\left(Q \perp h^{n}\right)^{m-1}\right)_{\pi_{m}} \otimes_{\left(A^{m-1}\right)_{\pi_{m}}[T]}\left(A_{\pi_{m}}^{m}[T]\right)\right)$ and $\left(\left(Q \perp h^{n}\right)^{m-1} \bigotimes_{A^{m-1}[T]} A^{m}[T]\right)_{\pi_{m}}$ with $\left(\left(Q \perp h^{n}\right)^{m-1} \bigotimes_{A^{m-1}[T]}\left(\left(A^{m}\right)_{\pi_{m}}[T]\right)\right)$, via the patching for quadratic spaces from [9], we have maps $\widetilde{\sigma}, \widetilde{\tau}$ corresponding to $\sigma, \tau$ and

$$
\widetilde{\sigma} \widetilde{\tau}^{-1} \in \varnothing_{\left(A^{m}\right)_{\pi_{m}[T]}}\left(\left(Q_{1} \perp h^{n}\right)^{m}{ }_{\pi_{m}}\right) .
$$

Since $\left(\left(A^{m}\right)_{\pi_{m}}\right)_{\mathfrak{m}}$ is local, $\left(\left(Q_{1}\right)^{m}{ }_{\pi_{m}}\right)_{\mathfrak{m}}$ is diagonalizable and hence, by Theorem [5.8,

$$
\varnothing\left(\left(\left(Q_{1}{ }^{m}\right)_{\pi_{m}}\right)_{\mathfrak{m}} \perp h^{n}\right)=\mathrm{EO}\left(\left(\left(Q_{1}{ }^{m}\right)_{\pi_{m}}\right)_{\mathfrak{m}} \perp h^{n}\right) \cdot \varnothing\left(h^{n}\right) .
$$

Therefore we can write

$$
\left(\widetilde{\sigma} \widetilde{\tau}^{-1}\right)_{\mathfrak{m}}=\alpha_{\mathfrak{m}} \beta_{\mathfrak{m}},
$$

where $\alpha_{\mathfrak{m}} \in \mathrm{EO}_{\left(\left(A^{m}\right)_{\pi_{m}}\right)_{\mathfrak{m}}[T]}\left(\left(\left(Q_{1}{ }^{m}\right)_{\pi_{m}}\right)_{\mathfrak{m}} \perp h^{n}\right)$ for some $\alpha \in \varnothing_{\left(A^{m}\right)_{\pi_{m}}[T]}\left(\left(\left(Q_{1}{ }^{m}\right)_{\pi_{m}}\right) \perp h^{n}\right)$ with $\alpha(0)=I d$ and $\beta_{\mathfrak{m}} \in \varnothing_{\left(\left(A^{m}\right)_{\pi_{m}}\right)_{\mathfrak{m}}[T]}\left(h^{n}\right)$ for some $\beta \in \varnothing_{\left(A^{m}\right)_{\pi_{m}}[T]}\left(h^{n}\right)$ with $\beta(0)=I d$, via the same argument as in Lemma [5.9.

Then, by Theorem 5.10, we have

$$
\widetilde{\sigma} \widetilde{\tau}^{-1}=\alpha \beta
$$

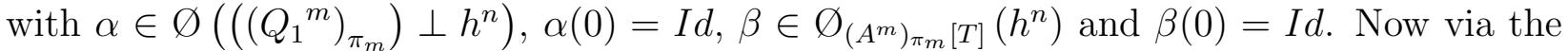
'deep splitting' technique introduced in [13], we can write $\widetilde{\sigma} \widetilde{\tau}^{-1}=\beta \in \varnothing\left(h^{n}\right)$.

Now we have

$$
\begin{aligned}
\left(Q \perp h^{n}\right)^{m-1} & \simeq\left(\left(\left(Q \perp h^{n}\right)^{m-1}\right)_{\pi_{m}}, I d,\left(Q \perp h^{n}\right)^{m}\right) \\
& \simeq\left(\left(A^{m-1}\right)_{\pi_{m}}[T] \otimes\left(\left(Q_{1}{ }^{m-1}\right)_{\pi_{m}} \perp h^{n}\right), \alpha \beta, A^{m}[T] \otimes\left(Q_{1}{ }^{m} \perp h^{n}\right)\right) \\
& \simeq\left(Q_{1}{ }^{m-1} \pi_{m}[T] \perp h^{n}, \beta, Q_{1}{ }^{m}[T] \perp h^{n}\right) \\
& \simeq Q_{1}{ }^{m-1}[T] \perp\left(h^{n}, \beta, h^{n}\right)=Q_{1}{ }^{m-1}[T] \perp Q_{2},
\end{aligned}
$$


where $Q_{2}$ is the quadratic $A^{m-1}[T]$-space defined by the patching technique. Now

$$
Q_{1}{ }^{m-1}[T] \perp Q_{2} \perp h^{r} \simeq Q^{m-1} \perp h^{r} \simeq Q_{1}{ }^{m-1}[T] \perp h^{n+r} .
$$

By cancellation of quadratic spaces over local rings [15], we have $Q_{2} \perp h \simeq h^{n+1}$. Since $\beta(0)=I d, Q_{2} /(T) \simeq h^{n}$. Thus, by Lemma 6.1, $Q_{2}$ is extended from $A^{m-1}$, whence so is $\left(Q \perp h^{n}\right)^{m-1}$. Hence the result is true for $l+1$. Then the theorem follows by induction.

Acknowledgements. The first named author is indebted to her advisor B. Sury for his support, unstinting help and encouragement during the course of this work. She is also thankful to the Tata Institute of Fundamental Research, Mumbai for their hospitality from time to time as this work progressed.

\section{References}

\section{References}

[1] A. A. Ambily, Yoga of commutators in Roy's elementary orthogonal transformation group, arXiv:1305.2826 [math.AC], 2013.

[2] R. Baeza, Quadratic forms over semilocal rings, Lecture Notes in Mathematics, Vol. 655, Springer-Verlag, Berlin, 1978.

[3] A. Bak, On modules with quadratic forms, in: Algebraic $K$-Theory and its Geometric Applications (Conf., Hull, 1969), Springer, Berlin, 1969, pp. 55-66.

[4] A. Bak, Nonabelian $K$-theory: the nilpotent class of $K_{1}$ and general stability, $K$-Theory 4 (4) (1991) 363-397.

[5] H. Bass, Unitary algebraic $K$-theory, in: Algebraic $K$-theory, III: Hermitian $K$-theory and geometric applications (Proc. Conf., Battelle Memorial Inst., Seattle, Wash., 1972), Lecture Notes in Math., Vol. 343, Springer, Berlin, 1973, pp. 57-265.

[6] I. Bertuccioni, A short proof of a theorem of Suslin-Kopelko, Arch. Math. (Basel) 39 (1) (1982) 9-10.

[7] A. J. Hahn, O. T. O'Meara, The classical groups and $K$-theory, vol. 291 of Grundlehren der Mathematischen Wissenschaften [Fundamental Principles of Mathematical Sciences] with a foreword by J. Dieudonné, Springer-Verlag, Berlin, 1989.

[8] T. Y. Lam, Serre's problem on projective modules, Springer Monographs in Mathematics, Springer-Verlag, Berlin, 2006.

[9] M. Ojanguren, Quadratic forms over regular rings, J. Indian Math. Soc. (N.S.) 44 (1-4) (1980) 109-116.

[10] B. Plumstead, The conjectures of Eisenbud and Evans, Amer. J. Math. 105 (6) (1983) 1417-1433.

[11] D. Popescu, General Néron desingularization, Nagoya Math. J. 100 (1985) 97-126.

[12] D. Quillen, Projective modules over polynomial rings, Invent. Math. 36 (1976) 167-171.

[13] R. Rao, Extendability of quadratic modules with sufficient Witt index, J. Algebra 86 (1) (1984) 159-180.

[14] R. A. Rao, Extendability of quadratic modules with sufficient Witt index. II, J. Algebra 89 (1) (1984) 88-101. 
[15] A. Roy, Cancellation of quadratic form over commutative rings, J. Algebra 10 (1968) 286-298.

[16] A. Roy, Application of patching diagrams to some questions about projective modules, J. Pure Appl. Algebra 24 (3) (1982) 313-319.

[17] V. Suresh, Linear relations in Eichler orthogonal transformations, J. Algebra 168 (3) (1994) 804-809.

[18] A. A. Suslin, The structure of the special linear group over rings of polynomials, Izv. Akad. Nauk SSSR Ser. Mat. 41 (2),477 (1977) 235-252.

[19] A. A. Suslin, V. I. Kopeiko, Quadratic modules and the orthogonal group over polynomial rings, Zap. Naučn. Sem. Leningrad. Otdel. Mat. Inst. Steklov. (LOMI) 71 (1977), 287, 216-250.

[20] G. Tang, Hermitian groups and $K$-theory, $K$-Theory 13 (3) (1998) 209-267.

[21] C. T. C. Wall, On the orthogonal groups of unimodular quadratic forms. II, J. Reine Angew. Math. 213 (1963/1964) 122-136. 